\title{
Ibuprofen Decreases Spontaneous Activity and Enhances Nerve-Evoked Contractions to Minimize Mitomycin C-Induced Bladder Dysfunction
}

\author{
Eliza G. West, Ryan Lang, Donna Sellers, Russ Chess-Williams, and \\ Catherine McDermott \\ Centre for Urology Research, Faculty of Health Sciences and Medicine, Bond University, Robina, Queensland, Australia
}

Received March 7, 2018; accepted May 17, 2018

\begin{abstract}
Inflammation may play a causal role in urological side effects reported following intravesical mitomycin $\mathrm{C}$ (MMC). Our aim was to investigate the effects of the nonsteroidal anti-inflammatory drug ibuprofen (IBU) on the cytotoxic potency of MMC and the potential for IBU to protect against bladder dysfunction. Malignant (RT4, T24) and normal (UROtsa) urothelial lines were treated with MMC followed by ibuprofen, with cell viability and caspase-3 activity assessed. Female C57BL/6JArc mice (Saline/Control, $\mathrm{MMC}$, Saline + IBU, and $\mathrm{MMC}+\mathrm{IBU})$ received intravesical treatment (1 hour) with saline or MMC $(2 \mathrm{mg} / \mathrm{ml})$, with IBU $(1 \mathrm{mg} / \mathrm{ml})$ delivered in drinking water (for 7 days). Voiding pattern analysis was conducted prior to and following (1, 3, 7 days) treatment. A whole-bladder preparation was used to assess compliance, contractile responses, and urothelial-mediator release. Ibuprofen
\end{abstract}

selectively increased the cytotoxic potency of $\mathrm{MMC}$ and caspase3 activity in both malignant cells lines but not in UROtsa. MMC significantly increased voiding frequency at 24 hours and 3 days, whereas administration of ibuprofen significantly reduced this effect. MMC significantly increased the frequency of spontaneous contractions from $2.3 \pm 0.5$ contractions $/ \mathrm{min}$ in saline controls to $4.8 \pm 0.16$ contractions $/ \mathrm{min}$, with ibuprofen protecting against this change. Interestingly, although nerve-evoked responses were not altered by MMC, they were increased in both IBU groups. Ibuprofen improved voiding dysfunction following MMC treatment by reducing spontaneous phasic activity and enhancing nervemediated contractions. Ibuprofen use in bladder cancer patients may help to minimize the urological adverse effects associated with intravesical MMC.

\section{Introduction}

Bladder cancer is a common malignancy worldwide, with most patients being diagnosed with the nonmuscle invasive form of the disease. Treatment generally begins with transurethral resection of the tumor, followed by intravesical instillation of a chemotherapeutic agent (Babjuk et al., 2011). Mitomycin $\mathrm{C}$ is the most common cytotoxic drug used to treat bladder cancer and has been shown to reduce the risk of recurrence (Bosschieter et al., 2018). Although it is an effective intravesical treatment, mitomycin C (MMC) is known to cause urological side effects in $34.5 \%-41 \%$ of patients who undergo treatment, the most common being increased urinary frequency and urgency, and painful urination (Thrasher and Crawford, 1992; Filson et al., 2014). Other side effects include pelvic pain and hematuria. In some cases, patients will not continue treatment because the side effects diminish their quality of life, which in turn has a substantial impact on treatment outcomes.

There is currently no clinically proven management strategy to help control the local adverse effects associated with intravesical treatment of bladder cancer. The muscarinic

https://doi.org/10.1124/jpet.118.248989. receptor antagonist oxybutynin has been reported to worsen the urological side effects reported by bladder cancer patients following intravesical immunotherapy with bacillus Calmette-Guérin (Johnson et al., 2013). Recently, Luckenbaugh et al. (2017) proposed the first management algorithm with an escalating pharmacological approach for managing MMCinduced cystitis in bladder cancer patients. This method included behavioral therapy initially followed by histamine antagonists, combined cholinergic and $\alpha$-adrenoceptor antagonist treatment, intensifying to prednisone combined with antihistamines in more severe cases. The authors acknowledge that this treatment approach has yet to be assessed in a formal clinical trial.

In vivo studies in rodents have reported alterations in bladder function that persist for several weeks following repeated intravesical treatment with MMC, epirubicin, or doxorubicin (Post et al., 1993; Michielsen et al., 2005). These changes include increased urinary frequency and decreased bladder compliance. In addition, MMC caused significantly more histologic damage to the mouse bladder compared with doxorubicin at clinically relevant doses (Post et al., 1993). Farr et al. (2017) recently reported that unlike gemcitabine which is selective for malignant over nonmalignant urothelial cells, MMC exhibited no such selectivity, with equivalent cytotoxic

ABBREVIATIONS: ACh, acetylcholine; ANOVA, analysis of variance; EFS, electrical field stimulation; IBU, ibuprofen; MMC, mitomycin C; NSAID, nonsteroidal anti-inflammatory drug; $\mathrm{PGE}_{2}$, prostaglandin $\mathrm{E}_{2}$. 
potency in normal and malignant bladder urothelial cultures. This may contribute to the more favorable efficacy and toxicity profile for gemcitabine compared with MMC (Shelley et al., 2012). Experimental studies have reported that doxorubicin, MMC, and gemcitabine, which are used for intravesical bladder cancer treatment, cause release of inflammatory cytokines, an effect that was still evident 1 week following treatment in in vitro urothelial models (Kang et al., 2013, 2015a; Farr et al., 2017). The findings of these studies suggest that inflammation may play a causal role in the urological side effects associated with intravesical chemotherapy and is therefore a potential target for treatment.

It is important to consider the impact that an adjunct antiinflammatory agent may have on the cytotoxic potency of intravesical chemotherapeutic agents such as MMC, and its efficacy as a bladder cancer treatment. Therefore, the first aim of this study was to investigate the effects of nonsteroidal antiinflammatory drug ibuprofen on the cytotoxic potency of MMC in bladder cancer and nonmalignant urothelial cells lines. Secondly, we aimed to assess the potential for ibuprofen to protect against bladder dysfunction in mice following intravesical MMC treatment.

\section{Methods}

\section{Materials}

Mitomycin C was purchased from Cayman Chemical Company (Ann Arbor, MI). Ibuprofen oral suspension $(200 \mathrm{mg} / \mathrm{ml}$ ) was prepared by National Custom Compounding, Merrimac, Australia. All chemicals used to prepare Krebs bicarbonate solution and saline, as well as carbachol, isoprenaline, ATP, $\alpha, \beta$-methylene-ATP, atropine, and $\mathrm{L}-N$-nitroarginine were purchased from MilliporeSigma (St. Louis, MO). The ATP Determination Kit and Amplex Red Acetylcholine Assay Kit were Molecular Probes brand (Thermo Fisher Scientific, Waltham, MA).

\section{Cell Culture}

Human RT4 and T24 bladder urothelial cancer cells (European Collection of Cell Cultures) were cultured in McCoy's 5A culture medium, supplemented with $10 \%$ fetal bovine serum and L-glutamine and gentamicin. Normal urothelial cells (UROtsa; a gift from Professor S. Garrett, University of North Dakota) were cultured and maintained in low-glucose Dulbecco's modified Eagle's medium, supplemented with $5 \%$ fetal bovine serum and gentamicin. Fresh culture medium was added every 2 days and cells were maintained at $37^{\circ} \mathrm{C}$ in a $5 \% \mathrm{CO}_{2}$ incubator. Cells were split when they reached approximately $90 \%$ confluence. Trypan blue exclusion was used to assess cell viability prior to plating for experiments. Only cell cultures with $\geq 95 \%$ viability were used.

\section{Cell Treatment}

Ninety-six-well plates were seeded at a density of 50,000 cells/well and incubated overnight at $37^{\circ} \mathrm{C}$ in a $5 \% \mathrm{CO}_{2}$ incubator to allow cells to attach. Following incubation, culture medium was removed and replaced with fresh culture medium containing MMC (0.13-1.3 mM). Untreated and vehicle-treated controls were included in each experiment. Cells were incubated for 2 hours with MMC to mimic maximum duration of intravesical treatment, at which time treatment medium was removed. Cells were washed twice with phosphate-buffered saline and fresh culture medium added to each of the wells. Cells were maintained in culture for a further 24 or 72 hours, at which time apoptosis and cell viability was assessed.
To determine the effects of the anti-inflammatory drug ibuprofen on the cytotoxic potency of MMC, cells were treated as described above, but following treatment, medium containing ibuprofen $(5 \mu \mathrm{M})$ was added to the cells and then incubated for a 24- or 72-hour recovery period in the presence of the anti-inflammatory drug. Appropriate vehicle controls were included in each experiment. Each condition was tested in triplicate wells, with a minimum of $n=5$ repeat experiments conducted.

\section{Measurement of Cell Viability and Caspase-3 Activity}

The resazurin reduction assay, whereby resazurin is reduced to resorufin by viable cells, was used to assess cell viability. Seventytwo hours after MMC treatment, the medium was removed from the 96-well plate and fresh medium containing $44 \mu \mathrm{M}$ resazurin was added. After a 2-hour incubation, reduction of resazurin to resorufin was determined by fluorescence (excitation $530 \mathrm{~nm}$, emission $590 \mathrm{~nm}$ ) using a Modulus microplate reader (Promega Corporation, Madison, WI). Under all conditions tested, the extent of resazurin reduction was directly proportional to viable cell counts (data not shown).

Caspase-3 activity was assessed in cells treated with MMC alone or in combination with the anti-inflammatory drug as a marker of early apoptosis. Caspase-3 activity was assessed at 24 hours post-treatment using a Caspase-3 Fluorescence Assay Kit (Cayman Chemical). All steps followed the manufacturer's instructions. Caspase-3 data were normalized according to cell number using corresponding resazurin reduction data.

\section{Animal Model}

All procedures were performed in accordance with the Australian Code for the Care and Use of Animals for Scientific Purposes and with the approval of the University of Queensland Animal Ethics Committee. Adult female C57BL/6JArc mice (32 weeks in age) were used for this study and housed under environmentally controlled conditions, with 12-hour light-dark cycles and access to food and water ab libitum. Animals were randomly allocated into four experimental groups: Saline, MMC, Saline + IBU or MMC + IBU.

\section{Intravesical Treatment}

Mice were anesthetized with 1\%-3\% isoflurane gas and micturition was induced by mild abdominal massage to prevent dilution of solutions. Bladder catheterization was then performed with a sterile intravenous $22 \times 1$-gauge catheter (Terumo Corporation, Tokyo, Japan) through the urethra. Thirty microliters of solution (saline or $1 \mathrm{mg} / \mathrm{ml}$ MMC, clinical dose) was instilled through the catheter before removal. Mice were allowed to run freely in their cage with access to food, but not water, for 1 hour on $2-\mu \mathrm{m}$ retention Hardened Ashless Filter Paper-225 (Filtech Pty Ltd, Wollongong, Australia), ensuring they did not void during this treatment period. Micturition was induced 1 hour postintravesical instillation to expel the drug or saline solution.

\section{Ibuprofen Treatment}

Ibuprofen was chosen on the basis of initial anti-inflammatory screening in the malignant and nonmalignant urothelial culture models (see Results and Discussion). To determine the effects of ibuprofen on bladder dysfunction caused by intravesical MMC treatment, mice received $1 \mathrm{mg} / \mathrm{ml}$ ibuprofen (Bosgraaf et al., 2006) in their drinking water for 7 days following intravesical treatment with saline or MMC. These animals were compared with the saline or MMC only groups.

\section{Voiding Pattern Analysis}

Voiding analysis was performed on the mice before intravesical treatment and was repeated 24 hours, and 3 and 7 days after treatment, 
A

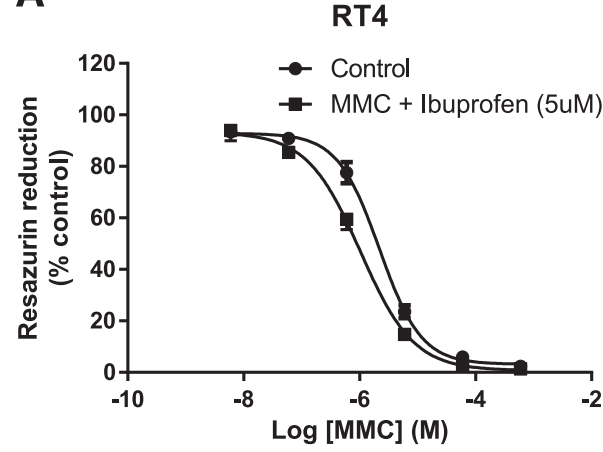

C UROtsa

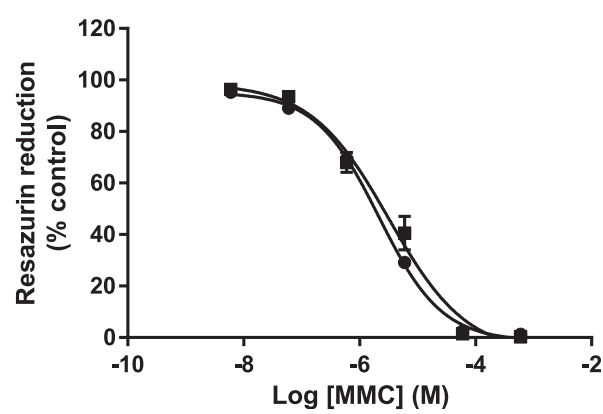

B

T24

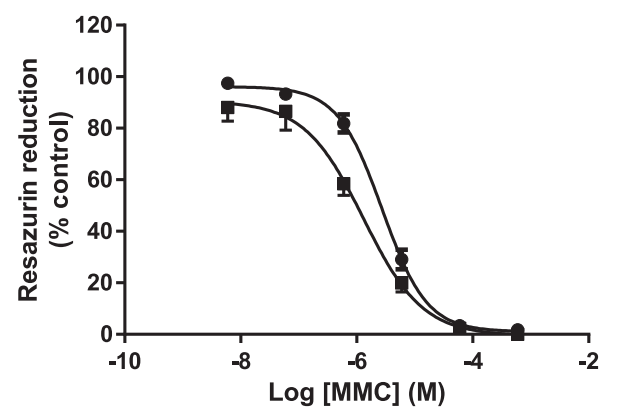

D

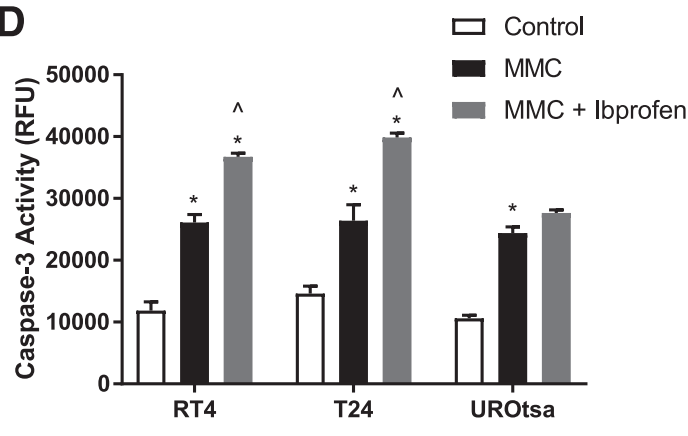

Fig. 1. Effect of MMC and ibuprofen on cell proliferation in (A) RT4, (B) T24, and (C) UROtsa cells measured as resazurin reduction 72 hours following 2-hour MMC treatment, and (D) caspase-3 activity at 24 hours. Data represents mean \pm S.E.M. $(n=5)$. Data analyzed using one-way ANOVA with Tukey-Kramer multiple comparisons test (* vs. Control; ^ vs. MMC).

before euthanasia. All voiding pattern analysis was performed in the morning, beginning at the change-over of the light/dark cycle. Mice were housed singly in cages lined with Filtech Hardened Ashless Filter Paper no. 225 for 4 hours with free access to food and water. The filter paper was collected, and urine spots detected using a Molecular Imager ChemiDoc XRS UV transilluminator (cat. no. 720BR1293; BioRad, Hercules, CA). The papers were photographed, digitized, and analyzed using Image J software. Voided volume was determined using a standard curve created by measuring the area of urine patches of known volume $(1-200 \mu \mathrm{l})$ and analyzed using linear regression analysis $\left(r^{2}=0.997\right)$. The basis of this protocol were previously reported studies of the voiding patterns of mice (Boudes et al., 2011; Uvin et al., 2013).

Isolated Whole Bladder Preparation. Mice were euthanized by cervical dislocation 7 days postintravesical treatment. The bladder was then isolated and a three-way cannula inserted via the urethra to enable recording of intravesical pressure in addition to bladder filling and emptying, and mounted into a modified tissue bath $(8 \mathrm{ml})$ containing gassed $\left(95 \% \mathrm{O}_{2} / 5 \% \mathrm{CO}_{2}\right)$ Krebs bicarbonate solution (composition in $\mathrm{mM}$ : $\mathrm{NaCl} 118$, NaHCO3 24.9, CaCl2 1.9, MgSO4 1.15, KCl 4.7, KH2PO4 1.15, and D-glucose 11.7$)$ at $37^{\circ} \mathrm{C}$. Intravesical pressure was measured using a pressure transducer (obtained from GlobalTown Microtech, Sarasota, FL) connected to a PC via a PowerLab data acquisition system (AD Instruments, Sydney, Australia), using LabChart 7 software (AD Instruments).

Following equilibration, bladder distensions were performed by intravesical infusion of isotonic saline at a rate of $30 \mu \mathrm{l} / \mathrm{min}$ to $40 \mathrm{mmHg}$ to check viability and compliance, and to $20 \mathrm{mmHg}$ for all further distensions for experimental purposes.

The urothelium is known to play a signaling role, releasing several chemical mediators in response to distension during bladder filling, these include ATP and acetylcholine (ACh). Following distension to $20 \mathrm{mmHg}$ the bladder was drained via the twoway cannula and intraluminal contents collected for measurement of the urothelial mediators ATP and ACh. Samples of the serosal fluid were also collected, and all samples were immediately frozen on dry ice and stored at $-80^{\circ} \mathrm{C}$ for later assay of ATP and ACh, using the ATP Determination Kit (Molecular Probes), and the Acetylcholine Amplex Red Assay Kit (Molecular Probes). The assays were performed according to manufacturer instructions, and luminescence, fluorescence (excitation 540, emission $590 \mathrm{~nm}$ ), or absorbance measured, using a Modulus micro-plate reader (Promega).

Following distension to $20 \mathrm{mmHg}$, bladders were allowed to equilibrate. During this period of accommodation spontaneous activity was measured as frequency of spontaneous contractions, recorded as number of contractions per minute, and amplitude measured from the peak to the trough of the contraction.

Electrical field stimulation (EFS) was undertaken in bladders after a 1-hour equilibration following distension to $20 \mathrm{mmHg}$. The bladder was electrically stimulated ( $50 \mathrm{~V})$, every 100 seconds at 1,5 , 10 , and $20 \mathrm{~Hz}$, and contractions were measured as pressure change from baseline. EFS was then repeated at $20 \mathrm{~Hz}$ in the presence of L-NNA $(100 \mu \mathrm{M})$, atropine $(1 \mu \mathrm{M})$, and $\alpha, \beta$-methylene ATP $(10 \mu \mathrm{M})$. Neurogenic origins of the pressure response to EFS were confirmed using tetrodotoxin $(0.1 \mu \mathrm{M})$, which abolished responses at all frequencies.

Pressure responses to pharmacological agents was also assessed by addition of ATP $(10 \mathrm{mM})$, cumulative carbachol concentrations, and relaxation to cumulative isoprenaline concentrations. Finally, $60 \mathrm{mM} \mathrm{KCl}$ solution was added to measure nonreceptor-mediated contractile response in the whole bladder. All contractions and relaxation responses were measured as a change in pressure from baseline.

Data and Statistical Analysis. All experiments were randomized, with five mice per experimental group and each experimental protocol started on a different day. For cell culture experiments five independent experiments were conducted in triplicate as technical replicates to ensure reliability of single values. The data 

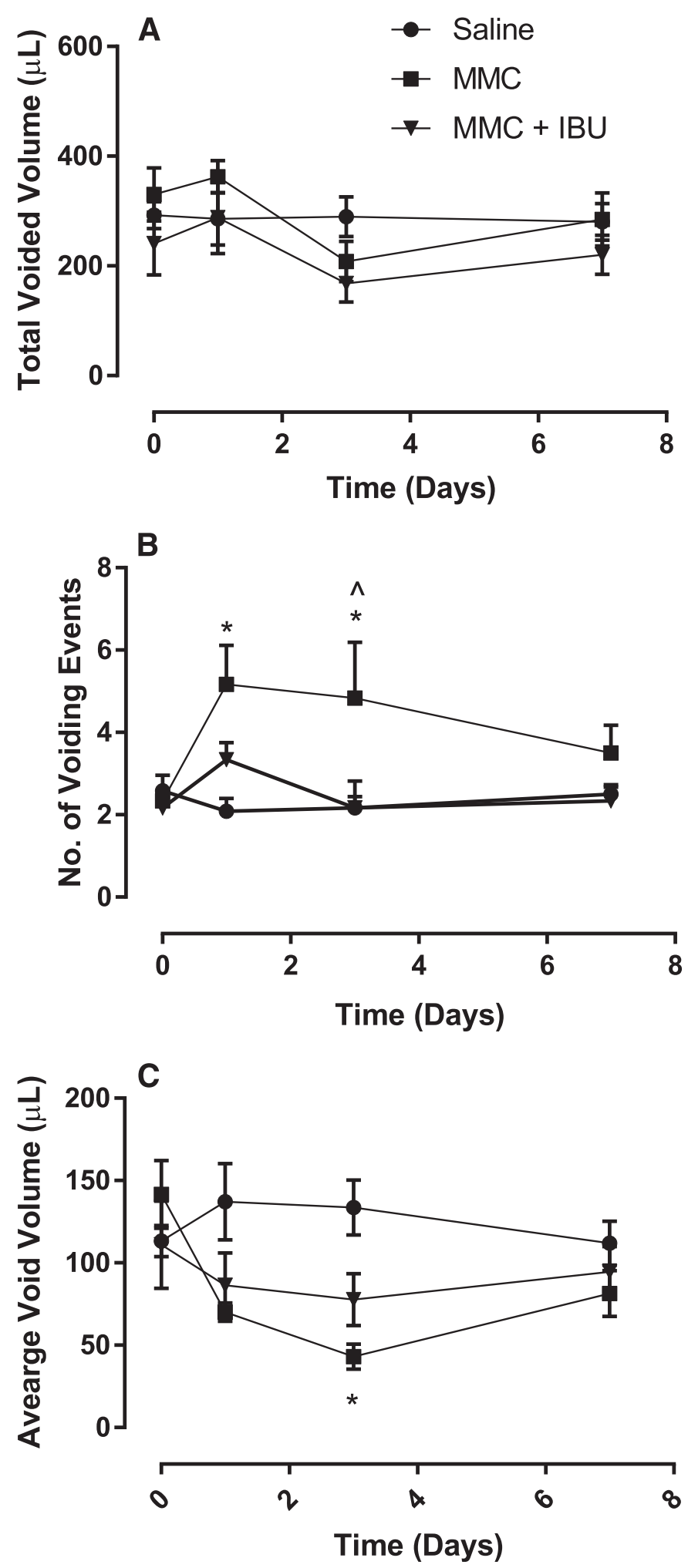

Fig. 2. Voiding pattern analysis conducted in mice from Control/Saline, MMC, and MMC + IBU groups measuring (A) total voided area (square centimeters), (B) number of voiding events, (C) average urine spot size. Data are presented as mean \pm S.E.M. $(n=6)$ and analyzed using repeated measures two-way ANOVA with significant values indicated $\left({ }^{*} P<0.05\right.$, MMC vs. Saline; ${ }^{\wedge} P<0.01, \mathrm{MMC}$ vs. MMC + IBU).

presented for cell culture experiments represent the mean of $n=$ 5 single values. Results are expressed as mean \pm S.E.M. Data were analyzed using a unpaired Student $t$ test, ordinary one-way analysis of variance (ANOVA) or repeated measures two-way ANOVA with Bonferroni multiple comparisons test, using GraphPad Prism version 6 software (GraphPad, La Jolla, CA). Significance levels were defined as $P<0.05$ (*).

\section{Results}

Effect of MMC on Malignant and Nonmalignant Urothelial Cell Lines. Two-hour treatment with MMC resulted in a concentration-dependent decrease in cell viability as measured by resazurin reduction in RT4, T24 and UROtsa (Fig. 1, A-C), assessed 72 hours following treatment. MMC was not selective for malignant cell lines (RT4 and T24) over the nonmalignant UROtsa, with equivalent cytotoxic potency observed in all of the urothelial cell lines tested $\left(\mathrm{pIC}_{50}\right.$ : RT4 $5.66 \pm 0.05$, T24 $5.67 \pm 0.03$, UROtsa $5.67 \pm 0.05$ ). Ibuprofen did not reduce the cytotoxic potency of MMC but did selectively and significantly increase the cytotoxic potency of MMC in both malignant urothelial cell lines $\left(\mathrm{pIC}_{50} \mathrm{RT} 4: 5.66\right.$ $\pm 0.05 \mathrm{MMC}$ alone vs. $5.99 \pm 0.06 \mathrm{MMC}+\mathrm{IBU} P<0.01 ; \mathrm{pIC}_{50}$ T24: $5.67 \pm 0.03 \mathrm{MMC}$ alone vs. $5.89 \pm 0.12 \mathrm{MMC}+\mathrm{IBU} P<$ 0.05 ), with no effect on the potency in the nonmalignant UROtsa cell line (Fig. 1, A-C). The increase in cytotoxic potency of MMC when combined with ibuprofen was associated with a significant increase in activity of the apoptotic marker caspase-3 in both RT4 $(P<0.01)$ and T24 $(P<0.01)$ cells (Fig. 1D).

Effects of MMC and Ibuprofen on Animal Parameters and Voiding Pattern. Animal body weight and water consumption were measured prior to and at 1,3 , and 7 days following intravesical instillation. These parameters were not significantly affected by MMC or ibuprofen treatment (data not shown).

Voiding pattern data were collected from animals in all four experimental groups, but statistical analysis revealed no significant difference in voiding pattern between the Saline and Saline + IBU groups, indicating ibuprofen did not affect voiding behavior. As a result, the voiding pattern analysis data were combined for Saline and Saline + IBU groups and these combined data are presented graphically as the Saline group (Fig. 2). Overall there was no change in total urine volume voided over time or between experimental groups, indicating that rate of urine production was not affected by treatment with MMC or ibuprofen (Fig. 2A). Intravesical MMC significantly increased the number of voiding events compared with saline, 24 hours $(P<0.001)$ and 3 days $(P<0.01)$ following treatment (Fig. 2B) indicating increased frequency of urination. Although the number of voiding events was still elevated at 7 days following MMC treatment, the increase was not statistically significant. Ibuprofen attenuated the change in voiding frequency caused by intravesical MMC treatment, with significantly fewer voiding events $(P<0.05)$ at 3 days compared with the MMC-only group (Fig. 2B). The number of voiding events in the MMC + IBU group was then not significantly different from the saline group at any time point.

The increase in voiding frequency observed with MMC was accompanied by a decrease in the average volume of each void (spot size) in the MMC group 1 day $(P>0.05)$ and 3 days $(P<$ 0.05 ) following treatment, indicating a decreased volume of urine expelled with each void. While the MMC + IBU treatment group showed the same trend of decreasing average volume voided 24 hours after treatment, unlike the MMC-only group, the MMC + IBU group began to recover at 3 days (Fig. $2 \mathrm{C}$ ). 
TABLE 1

Whole-bladder responses from saline-, MMC-, saline + ibuprofen-, and MMC + ibuprofen-treated mice: filling volume at $40 \mathrm{mmHg}$ pressure, pressure response to ATP $(10 \mathrm{mM})$ and $\mathrm{KCl}(60 \mathrm{mM}), \mathrm{pEC}_{50}$ and maximal responses (millimeter of mercury) to carbachol and $\mathrm{pIC}_{50}$, and maximal responses to isoprenaline

Data presented are mean \pm S.E.M. $(n=6)$ analyzed using one-way ANOVA with Bonferroni multiple comparisons test.

\begin{tabular}{|c|c|c|c|c|}
\hline & Saline & MMC & Saline + IBU & $\mathrm{MMC}+\mathrm{IBU}$ \\
\hline Filling volume at $40 \mathrm{mmHg}(\mu \mathrm{l})$ & $481 \pm 74.6$ & $513 \pm 68.1$ & $487 \pm 40.6$ & $513 \pm 28.1$ \\
\hline \multicolumn{5}{|l|}{ Pressure Response } \\
\hline ATP (1 mM) (mmHg) & $1.11 \pm 0.29$ & $2.39 \pm 0.67$ & $2.14 \pm 0.67$ & $1.87 \pm 0.37$ \\
\hline $\mathrm{KCl}(60 \mathrm{mM})(\mathrm{mmHg})$ & $16.8 \pm 4.79$ & $10.04 \pm 3.55$ & $13.58 \pm 1.52$ & $13.32 \pm 4.03$ \\
\hline \multicolumn{5}{|l|}{ Carbachol } \\
\hline $\mathrm{pEC}_{50}$ & $5.85 \pm 0.22$ & $5.85 \pm 0.27$ & $6.05 \pm 0.12$ & $6.03 \pm 0.16$ \\
\hline Maximal response (mmHg) & $28.44 \pm 6.36$ & $23.69 \pm 6.09$ & $29.45 \pm 3.32$ & $28.82 \pm 4.56$ \\
\hline \multicolumn{5}{|l|}{ Isoprenaline } \\
\hline $\mathrm{pIC}_{50}$ & $7.03 \pm 0.33$ & $7.46 \pm 0.56$ & $7.29 \pm 0.22$ & $7.05 \pm 0.35$ \\
\hline Maximal response (mmHg) & $2.56 \pm 0.39$ & $1.92 \pm 0.74$ & $2.65 \pm 0.35$ & $2.26 \pm 0.38$ \\
\hline
\end{tabular}

Effects of MMC and Ibuprofen on Compliance and Contractile Responses of Isolated Whole Bladders. No significant difference in bladder compliance curves (data not shown) or bladder capacity (Table 1) was seen between treatment groups. Likewise, intravesical pressure responses to carbachol, ATP, $\mathrm{KCl}$, and isoprenaline were not significantly affected by intravesical MMC or ibuprofen administration (Table 1).

Spontaneous phasic contractions were observed in bladders from all treatment groups, with the amplitude and frequency of spontaneous contractions quantified during accommodation following distension to $20 \mathrm{mmHg}$. The amplitude of the spontaneous contractions in the saline group was $0.18 \pm$ $0.04 \mathrm{mmHg}$ and increased approximately 2 -fold in the MMC treatment group, although the change was not statistically significant $(P>0.05)$ (Fig. 3). There was a significant 2 -fold increase $(P<0.001)$ in the frequency of spontaneous contractions in the MMC group (4.8 \pm 0.16 contractions $/ \mathrm{min})$ compared with the saline control ( $2.3 \pm 0.5$ contractions $/ \mathrm{min})$ (Fig. 3B). Ibuprofen alone did not alter the frequency of spontaneous contractions, with no significant difference observed between the Saline and Saline + IBU groups. However, ibuprofen protected against the MMC-induced changes in frequency of nonvoiding contractions, with a significant decrease in the frequency observed in the MMC + IBU treatment group compared with MMC alone $(P<0.05)$, and ibuprofen maintained the frequency of spontaneous contraction at the rate observed in the Saline control group (Fig. 3B).

Electrical field stimulation was performed to assess the effect of treatment on efferent nerve-mediated contraction of murine bladders. A frequency-dependent increase in intravesical pressure was observed in bladders from all animal groups (Fig. 4A). Efferent evoked-pressure responses were not altered in bladders from MMC-treated animals compared with the saline group (Fig. 4A). Interestingly, the pressure response to EFS was consistently increased at all frequencies in both the Saline + IBU and MMC + IBU groups compared with Saline or MMC alone. The pressure response at $20 \mathrm{~Hz}$ in the MMC + IBU group was significantly greater than in the MMC group $(P<0.05)$ (Fig. 4A). Pharmacological agents were added to the bath to determine the relative contribution of nitric oxide and muscarinic and P2X receptors to nerve-evoked responses in the murine bladder. Desensitization of P2X receptors with $\alpha, \beta$-methylene-ATP, significantly decreased nerve-evoked responses in all experimental groups, indicating that ATP is the main neurotransmitter involved in efferent responses in the murine bladder (Fig. 4B, Saline group), and this is not altered by intravesical treatment with MMC or treatment with ibuprofen (data not shown).

Effects of Intravesical MMC and Ibuprofen on Intraluminal and Serosal Release of ATP and Acetylcholine from Isolated Whole Bladders. The urothelium plays a sensory and signaling role through release of chemical mediators in response to stretch during bladder filling. Samples of intraluminal and serosal fluid were collected from bladders distended to $20 \mathrm{mmHg}$ and analyzed for the release of urothelial mediators ATP and Ach to determine if changes in their release contributed to MMC-induced voiding dysfunction in mice. Overall, there was greater release of both ATP and ACh into the serosal fluid than there was into the intraluminal fluid in all groups. Levels of intraluminal and serosal ATP and intraluminal ACh were not affected by MMC or ibuprofen treatment $(P>0.05)$ (Fig. 5). However, serosal ACh was significantly increased in the MMC treatment group $(P<0.05)$, an effect that was also evident in the MMC + IBU group $(P<0.001)$ (Fig. 5D). The serosal ACh release was not significantly different between the MMC and the MMC + IBU, indicating that ibuprofen did not alter the MMCinduced changes in serosal ACh release. Additionally, ibuprofen did not alter serosal ACh in saline treated mice (Fig. 5D).

\section{Discussion}

There is abundant clinical evidence that bladder cancer patients experience urological side effects following intravesical treatment with MMC and other cytotoxic and immune therapies. Currently, no recommended approach to management of these adverse effects is supported by evidence from clinical trials. Our research group has previously investigated the effects of intravesical agents, including doxorubicin, gemcitabine, and MMC, on various aspects of bladder function, and the results of these studies indicate that inflammation may play a causal role in the urological adverse effects experienced by patients following treatment (Kang et al., 2015a; Farr et al., 2017). Therefore, the aim of this study was first to determine if an anti-inflammatory drug affected the cytotoxic potency of MMC and if administration of the nonsteroidal anti-inflammatory drug (NSAID) ibuprofen for 7 days following intravesical MMC treatment would minimize the effects of MMC on voiding behavior and bladder function. 

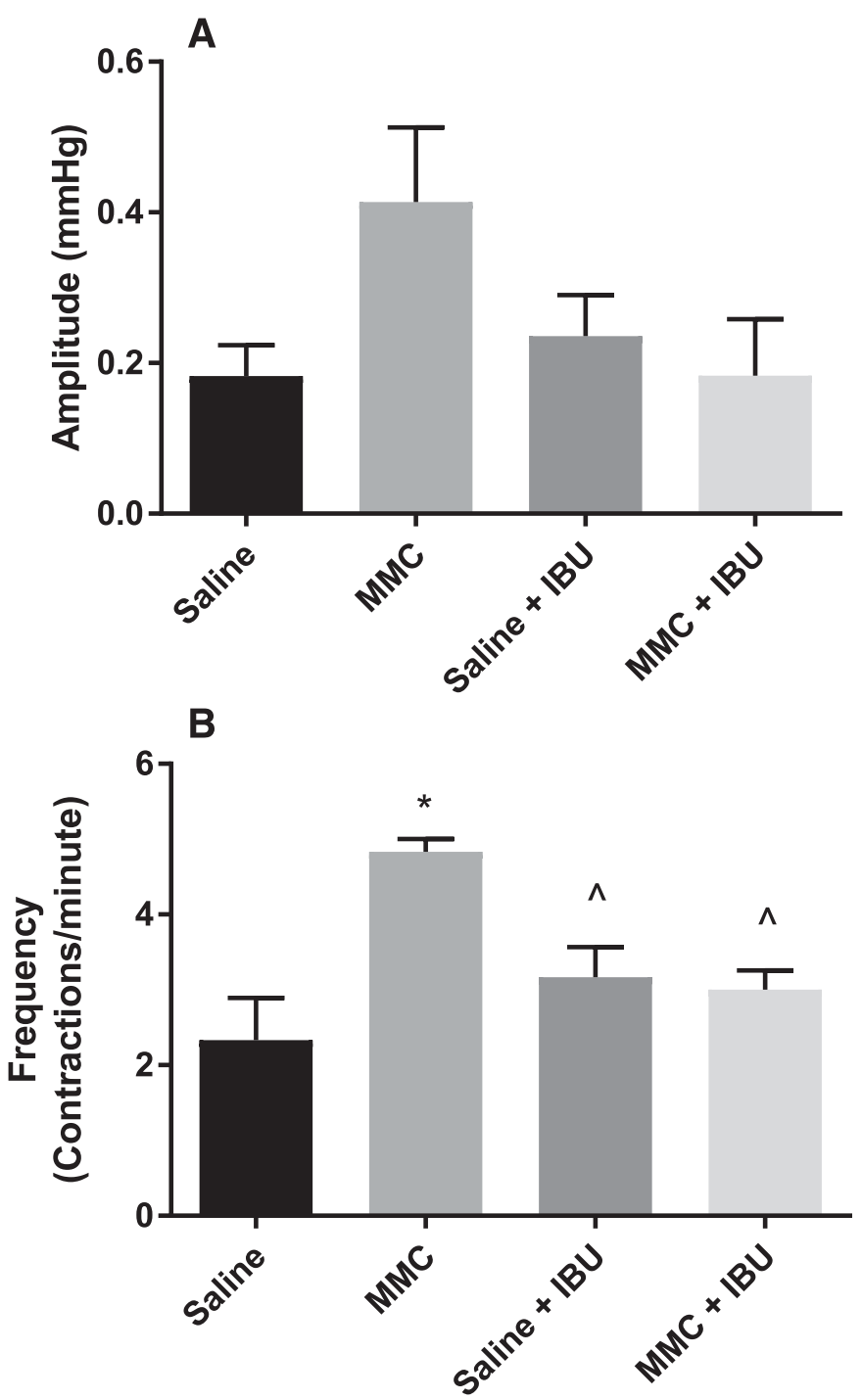

Fig. 3. Effect of MMC and ibuprofen treatments on spontaneous phasic contractions in the isolated murine bladder. (A) Amplitude of spontaneous contractions and (B) frequency of contractions. Data represented as mean \pm S.E.M. $(n=6)$, analyzed using one-way ANOVA with Bonferroni multiple comparisons test $(* P<0.001$, MMC vs. Saline) $(\wedge P<0.05$, MMC vs. Saline + IBU and MMC + IBU).

An important concern when considering adjunct therapies for cancer treatment is whether the drug combination will reduce the efficacy of the cancer treatment. Ibuprofen did not decrease the cytotoxicity of MMC in malignant or nonmalignant urothelial cultures. However, ibuprofen selectively and significantly enhanced the reduction in cell viability observed in MMC-treated bladder cancer cells but not in normal cell cultures. In addition, the activity of the apoptotic marker caspase-3 was also enhanced only in the cancer cell lines. This suggests that ibuprofen either enhances the cytotoxicity of MMC in malignant cells or has anticancer properties itself. A study previously examined the anticancer effects of NSAIDs on T24 and RT4 bladder cancer cell lines (Khwaja et al., 2004), including ibuprofen and indomethacin, and postulated that NSAIDs induce the expression of a tumor suppression gene, p75NTR. This gene was recently identified as a tumor suppressor and metastasis suppressor in bladder cancer cell lines (Krygier and Djakiew, 2001). Khwaja et al. (2004) found that ibuprofen treatment significantly caused a dosedependent expression of the p75NTR gene in both RT4 and T24 bladder cancer cell lines. Even though ibuprofen and indomethacin are both nonselective COX inhibitors and both induced a significant expression of the p75NTR gene, ibuprofen was more effective than indomethacin. This may explain why ibuprofen but not indomethacin (unpublished data) enhanced cytotoxicity and apoptosis in RT4 and T24 cells. Ibuprofen was chosen as the anti-inflammatory agent to use in the following animal study owing to its selective ability to increase the cytotoxic potency of MMC in bladder cancer cells.

The frequency of urination increased in MMC-treated mice, indicating development of an overactive bladder phenotype. This result was expected, as urinary frequency is one of the adverse effects reported by bladder cancer patients following intravesical chemotherapy (Alfred Witjes et al., 2008). Ibuprofen reduced the effect of MMC on urinary frequency, and although frequency was slightly increased $(P>0.05)$ at 24 hours, it returned to control levels at 3 days posttreatment, unlike the MMC group which did not show a significant decrease in the number of voiding events until 7 days. Overall, the increase in urinary frequency in MMC and MMC + IBU mice was associated with a decrease in average voided volume. Previously, our research group observed the effects of MMC ( $2 \mathrm{mg} / \mathrm{ml})$ on urothelial-mediator release and found that prostaglandin E2 was significantly increased 24 hours after treatment with MMC (Kang et al., 2015a). Prostaglandins are mediators of inflammation (Ricciotti and FitzGerald, 2011) and would be reduced by ibuprofen. It could then be concluded that by decreasing inflammation of the bladder, the increased urinary frequency caused by intravesical MMC was attenuated.

Many studies have observed spontaneous contractions in a number of different animal bladder models. Spontaneous activity can be characterized by either phasic or tonic contractions of the bladder. Contractions of the mouse urinary bladder occur mostly during the accommodation stage or bladder filling. These types of spontaneous contractions are called micromotions (Coolsaet, 1985). Here we observed that intravesical MMC resulted in an increase in the frequency and amplitude of spontaneous nonvoiding contractions measured 7 days following treatment. This increase in spontaneous activity may play a causal role in the increased urinary frequency observed in mice following MMC treatment. It has previously been reported that spontaneous contractions can initiate afferent nerve firing in a murine model of bladder overactivity (McCarthy et al., 2009). Early afferent activation owing to increased spontaneous activity could contribute to bladder overactivity in mice following MMC treatment. Prostaglandin rise in response to infection of the bladder or chemical cystitis (Grover et al., 2011) and have been shown to increase phasic contractions of detrusor smooth muscle (Kobayter et al., 2012). Prostaglandin $\mathrm{E}_{2}\left(\mathrm{PGE}_{2}\right)$ has the ability to increase the frequency of whole-cell $\mathrm{Ca}^{2+}$ transients and spontaneous depolarizations, thereby increasing spontaneous contractions. Kang et al. (2015a) found that MMC causes prostaglandin levels to rise 24 hours following treatment; therefore, the effect that MMC has on the bladder probably causes an inflammatory response.

Given the link between increased spontaneous activity and elevated prostaglandin release, use of cyclo-oxygenase inhibitors may minimize or eliminate changes in spontaneous 


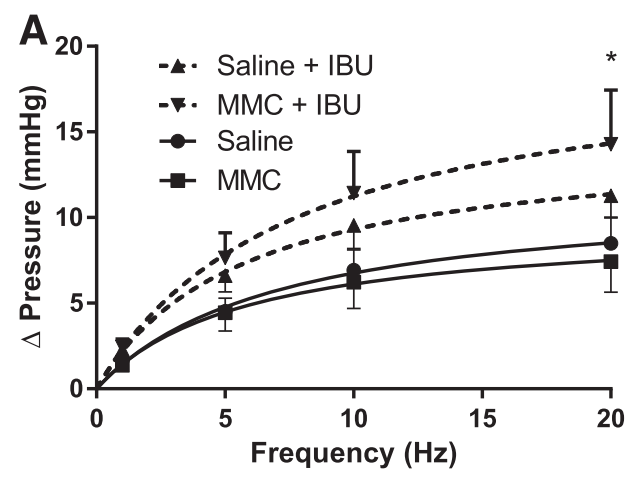

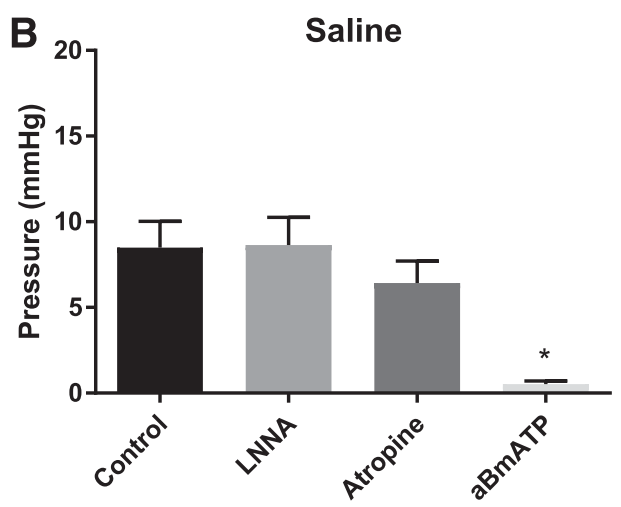

Fig. 4. (A) Nerve-evoked pressure responses in isolated bladders, from Saline, MMC, Saline + IBU and MMC + IBU groups to EFS at 1, 5, 10, and $20 \mathrm{~Hz}$. (B) Nerve-mediated contractions to EFS at $20 \mathrm{~Hz}$ in isolated bladders from Saline control group in the presence of LNNA $(100 \mu \mathrm{M})$, atropine $(1 \mu \mathrm{M})$ and $\alpha, \beta$-methylene-ATP $(10 \mu \mathrm{M})$. Responses were recorded as a change in pressure from baseline. Data are presented as the mean \pm S.E.M. $(n=6)$. Data analyzed using a (A) repeated measures two-way ANOVA and nonlinear regression (* vs MMC) or (B) oneway ANOVA with a Dunnett multiple comparisons test. aBmATP, $\alpha, \beta$-methylene-ATP (*vs all groups). activity caused by $\mathrm{PGE}_{2}$. Collins et al. (2009) hypothesized that prostaglandins may be released by interstitial cells of Cajal in the bladder and found that nonselective COX inhibitors reduced spontaneous contractions significantly. In the current study, the frequency of spontaneous contractions was significantly reduced by ibuprofen. Thus, development of an overactive bladder phenotype in mice treated with MMC may be explained by an increase in spontaneous activity via prostaglandin production.

Any dysfunction in afferent or efferent innervation probably results in inadequate emptying of the bladder on urination. Therefore, the increased urinary frequency in mice may result from sensitization of bladder sensory nerves or changes in efferent nerve activity. Bladder dysfunction may also arise from alterations in detrusor contractile responses or urothelial function. The ex vivo whole-bladder preparation was used to assess how MMC treatment affects bladder function. Responses to the pharmacological agents carbachol, ATP, and isoprenaline were not affected by treatment with MMC, nor were nerve-evoked responses. However, bladder responses to EFS were increased at all frequencies in both the Saline and MMC groups treated with ibuprofen. This indicates that ibuprofen increased the intravesical pressure generated during voiding contractions and probably contributed to the smaller change in urinary frequency observed in the MMC + IBU group compared with MMC alone. The reason for this increased contraction is not entirely clear; however, studies have found that prostaglandins may be key modulators in the
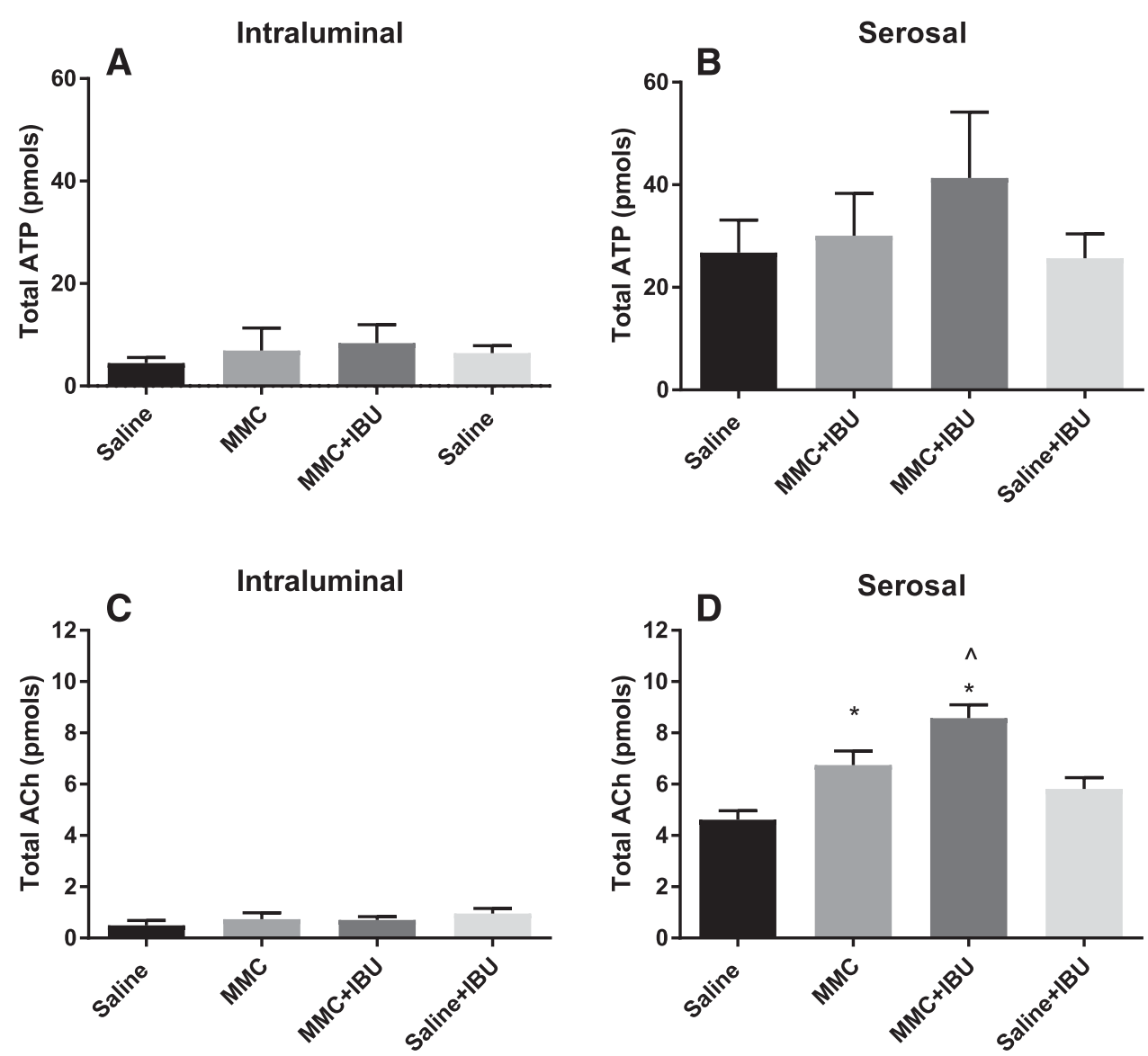

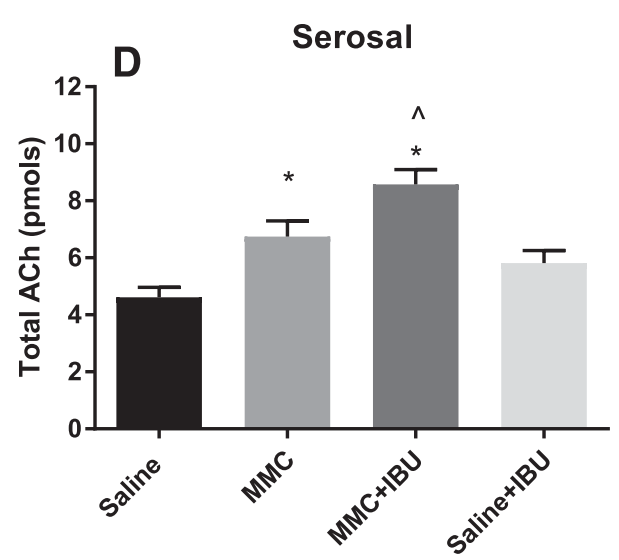

Fig. 5. Effect of intravesical MMC treatment and the effects of ibuprofen on the release of ATP and $\mathrm{ACh}$, from isolated bladders in response to distension with saline to $20 \mathrm{mmHg}$. (A and C) Intraluminal release and (B and $\mathrm{D})$ release into the serosal fluid. Data presented are mean \pm S.E.M. $(n=6)$ and analyzed using one-way ANOVA with Bonferroni multiple comparisons test (*vs. Saline, ${ }^{\wedge}$ vs. Saline + IBU). 
release of urothelial mediators during cystitis (Tessier et al., 1991; Senbel et al., 2017). Consistent with this idea, $\mathrm{PGE}_{2}$ has been reported to inhibit EFS-evoked contraction in rat bladders by activating cAMP-dependent $\mathrm{K}+$ channels (Ruan et al., 2008). As MMC is known to cause cystitis involving production of $\mathrm{PGE}_{2}$, it may be concluded that ibuprofen acts to minimize $\mathrm{PGE}_{2}$ synthesis, thereby increasing EFS contractions, thus enhancing voiding contractions and reducing residual volume, and therefore voiding frequency (Pessina et al., 2001). Residual urine volume is linked to urinary frequency and urinary incontinence (Khandelwal and Kistler, 2013), which is one of many adverse effects associated with MMC treatment (Alfred Witjes et al., 2008). Although MMC alone did not alter contractile responses to EFS, this may be attributable to concurrent release following intravesical MMC of numerous other inflammatory mediators that are not cyclo-oxygenase products. One such example is the inflammatory mediator bradykinin, which has been shown to enhance electrically evoked contractions in the mouse bladder (Fabiyi and Brading, 2006).

Previous studies have reported histologic changes to the urothelium following intravesical chemotherapy; this is not surprising given the intimate contact between the urothelium and high concentrations of cytotoxic agents (Becopoulos et al., 1991; Hou et al., 2011; Castillo et al., 2012). Here we assessed changes in urothelial function through measurement of the urothelial mediators ATP and Ach, which are known to regulate bladder function. There was no significant change in release of ATP across the treatment groups at 7 days, indicating that changes in ATP do not contribute to the voiding dysfunction evident in mice treated with MMC. Previously, we have shown that the intravesical agent doxorubicin increased release of ATP in treated porcine bladder immediately following treatment (Kang et al., 2015b). The changes in urothelial-mediator release, however, may be transient, as seen in urothelial cultures treated with MMC (Kang et al., 2015a). Serosal release of ACh was significantly increased in the MMC and MMC + IBO treatment groups, compared with the control group. Since ACh remained elevated in the MMC + IBO group it is improbable that this plays a substantial role in the voiding dysfunction observed in MMC-treated mice. Although the actions of urothelial Ach are not fully understood, it is known that ACh can act in two ways; to contract the detrusor muscle by acting on muscarinic receptors or by acting on afferent nerve terminals altering bladder sensation (Daly et al., 2007).

Animal behavior was monitored over the 7 days following treatment, in line with ethical practice. Although not an intended outcome measure, it was observed that MMC treatment did cause lethargy in the mice 24 hours after treatment with reduced locomotor activity noted; however, this recovered by 3 days following treatment. Reduction in locomotor activity levels is indicative of pain in mice (Burkholder et al., 2012), which may explain the lethargy observed in the MMC-treatment group, and was probably the result of the MMC treatment (Elmamoun et al., 2014). Animals treated with MMC and given ibuprofen in their drinking water also experienced this effect, although to a lesser degree, and were fully recovered 3 days post-treatment, suggesting ibuprofen reduced the pain caused by intravesical MMC treatment.

The results presented here indicate that MMC has a negative effect on bladder function and induces increased urinary frequency in mice following treatment. MMC increased the frequency of voiding, an effect that was associated with increased spontaneous contractions. Furthermore, this study has shown the benefits of ibuprofen, in conjunction with intravesical treatment, in decreasing spontaneous activity and voiding frequency and enhancing nerve-evoked response. In conclusion, the urological adverse effects caused by intravesical MMC can be reduced with the use of ibuprofen in standard treatment. This may bring relief to patients who undergo intravesical treatment and in turn improve their quality of life and ability to tolerate treatment.

\section{Authorship Contributions}

Participated in research design: Sellers, Chess-Williams, McDermott.

Conducted experiments: West, Lang.

Performed data analysis: West, Lang, McDermott.

Wrote or contributed to the writing of the manuscript: West, Lang, Sellers, Chess-Williams, McDermott.

\section{References}

Alfred Witjes J, Palou J, Soloway M, Lamm D, Brausi M, Roan Spermon J, Persad R, Buckley R, Akaza H, Colombel M, et al. (2008) Clinical practice recommendations for the prevention and management of intravesical therapy-associated adverse events. Eur Urol Suppl 7:667-674.

Babjuk M, Oosterlinck W, Sylvester R, Kaasinen E, Böhle A, Palou-Redorta J, and Rouprêt M; European Association of Urology (EAU) (2011) EAU guidelines on non-muscle-invasive urothelial carcinoma of the bladder, the 2011 update. Eur Urol 59:997-1008.

Becopoulos T, Kapralos P, Kouri E, Lambreli P, and Sofras F (1991) Histological changes of the bladder mucosa following the administration of BCG adriamycin and RO 10-9359. Int Urol Nephrol 23:553-557.

Bosgraaf CA, Johnston NA, and Toth LA (2006) Oral ibuprofen as an analgesic after abdominal surgery in mice. J Am Assoc Lab Anim Sci 45:117.

Bosschieter J, Nieuwenhuijzen JA, van Ginkel T, Vis AN, Witte B, Newling D, Beckers GMA, and van Moorselaar RJA (2018) Value of an immediate intravesical instillation of mitomycin $\mathrm{C}$ in patients with non-muscle-invasive bladder cancer: a prospective multicentre randomised study in 2243 patients. Eur Urol 73:226-232.

Boudes M, Uvin P, Kerselaers S, Vennekens R, Voets T, and De Ridder D (2011) Functional characterization of a chronic cyclophosphamide-induced overactive bladder model in mice. Neurourol Urodyn 30:1659-1665.

Burkholder T, Foltz C, Karlsson E, Linton CG, and Smith JM (2012) Health evaluation of experimental laboratory mice. Curr Protoc Mouse Biol 2:145-165.

Castillo OA, Landerer E, Feria-Flores M, Vidal-Mora I, and Franco C (2012) Histological changes due to intravesical instillation of mitomycin C. Arch Esp Urol 65: 578-582, discussion 582.

Collins C, Klausner AP, Herrick B, Koo HP, Miner AS, Henderson SC, and Ratz PH (2009) Potential for control of detrusor smooth muscle spontaneous rhythmic contraction by cyclooxygenase products released by interstitial cells of Cajal. J Cell Mol Med 13 (9B):3236-3250.

Coolsaet B (1985) Bladder compliance and detrusor activity during the collection phase. Neurourol Urodyn 4:263-273.

Daly D, Chess-Williams R, Chappie C, and Grundy D (2007) Endogenously released Ach alters bladder sensation in a mechanism involving both nicotinic and muscarinc receptors. Eur Urol Suppl 6:39.

Elmamoun MH, Christmas TJ, and Woodhouse CR (2014) Destruction of the bladder by single dose mitomycin C for low-stage transitional cell carcinoma (TCC)avoidance, recognition, management and consent. BJU Int 113 (5b):E34-E38.

Fabiyi AC and Brading AF (2006) The use of the isolated mouse whole bladder for investigating bladder overactivity. J Pharmacol Exp Ther 319:1386-1394.

Farr SE, Chess-Williams R, and McDermott CM (2017) Gemcitabine: selective cytotoxicity, induction of inflammation and effects on urothelial function. Toxicol Appl Pharmacol 316:1-9.

Filson CP, Montgomery JS, Dailey SM, Crossley HS, Lentz H, Tallman CT, He C, and Weizer AZ (2014) Complications associated with single-dose, perioperative mitomycin-C for patients undergoing bladder tumor resection. Urol Oncol 32:40. e1-40.e8.

Grover S, Srivastava A, Lee R, Tewari AK, and Te AE (2011) Role of inflammation in bladder function and interstitial cystitis. Ther Adv Urol 3:19-33.

Hou JC, Landas S, Wang CY, and Shapiro O (2011) Instillation of mitomycin C after transurethral resection of bladder cancer impairs wound healing: an animal model. Anticancer Res 31:929-932.

Johnson MH, Nepple KG, Peck V, Trinkaus K, Klim A, Sandhu GS, and Kibel AS (2013) Randomized controlled trial of oxybutynin extended release versus placebo for urinary symptoms during intravesical Bacillus Calmette-Guérin treatment. $J$ Urol 189:1268-1274.

Kang SH, Chess-Williams R, Anoopkumar-Dukie S, and McDermott C (2013) Induction of inflammatory cytokines and alteration of urothelial ATP, acetylcholine and prostaglandin E2 release by doxorubicin. Eur J Pharmacol 700:102-109.

Kang SH, Chess-Williams R, Anoopkumar-Dukie S, and McDermott C (2015a) Recovery of urothelial mediator release but prolonged elevations in interleukin-8 and 
nitric oxide secretion following mitomycin C treatment. Naunyn Schmiedebergs Arch Pharmacol 388:781-791.

Kang SH, McDermott C, Farr S, and Chess-Williams R (2015b) Enhanced urothelial ATP release and contraction following intravesical treatment with the cytotoxic drug, doxorubicin. Naunyn Schmiedebergs Arch Pharmacol 388:773-780.

Khandelwal C and Kistler C (2013) Diagnosis of urinary incontinence. Am Fam Physician 87:543-550.

Khwaja F, Allen J, Lynch J, Andrews P, and Djakiew D (2004) Ibuprofen inhibits survival of bladder cancer cells by induced expression of the p75NTR tumor suppressor protein. Cancer Res 64:6207-6213.

Kobayter S, Young JS, and Brain KL (2012) Prostaglandin E2 induces spontaneous rhythmic activity in mouse urinary bladder independently of efferent nerves. $\mathrm{Br} J$ Pharmacol 165:401-413.

Krygier S and Djakiew D (2001) Molecular characterization of the loss of p75(NTR expression in human prostate tumor cells. Mol Carcinog 31:46-55.

Luckenbaugh AN, Marks RM, Miller DC, Weizer AZ, Stoffel JT, and Montgomery JS (2017) A management algorithm for mitomycin C induced cystitis. Bladder Cancer 3:133-138.

McCarthy CJ, Zabbarova IV, Brumovsky PR, Roppolo JR, Gebhart GF, and Kanai AJ (2009) Spontaneous contractions evoke afferent nerve firing in mouse bladders with detrusor overactivity. J Urol 181:1459-1466.

Michielsen D, Amy JJ, Coomans D, Storme G, and Wyndaele JJ (2005) Mitomycin C and epirubicin: functional bladder damage in rats after repeat intravesical instillations. J Urol 173:2166-2170.

Pessina F, Marazova K, Kalfin R, Sgaragli G, Manganelli A, and Milenov K (2001) Mechanical response to electrical field stimulation of rat, guinea-pig, monkey and human detrusor muscle: a comparative study. Naunyn Schmiedebergs Arch Pharmacol 363:543-550.
Post JG, te Poele JA, Oussoren Y, and Stewart FA (1993) Bladder damage in mice after single and repeated intravesical instillations of mitomycin $\mathrm{C}$ or doxorubicin. $J$ Urol 150:1965-1969

Ricciotti E and FitzGerald GA (2011) Prostaglandins and inflammation. Arterioscler Thromb Vasc Biol 31:986-1000.

Ruan YC, Wang Z, Du JY, Zuo WL, Guo JH, Zhang J, Wu ZL, Wong HY, Chung YW, Chan HC, et al. (2008) Regulation of smooth muscle contractility by the epithelium in rat vas deferens: role of ATP-induced release of PGE2. J Physiol 586:4843-4857. Senbel AM, Bassiouni WM, and Daabees TT (2017) Effect of some prostanoids modulators and cyclooxygenase inhibitors on rat bladder detrusor muscle in normal and cyclophosphamide- induced cystitis. Clin Ther 39:e75.

Shelley MD, Jones G, Cleves A, Wilt TJ, Mason MD, and Kynaston HG (2012) Intravesical gemcitabine therapy for non-muscle invasive bladder cancer (NMIBC): a systematic review. BJU Int 109:496-505.

Tessier GJ, Lackner PA, O'Grady SM, and Kannan MS (1991) Modulation of equine tracheal smooth muscle contractility by epithelial-derived and cyclooxygenase metabolites. Respir Physiol 84:105-114.

Thrasher JB and Crawford ED (1992) Complications of intravesical chemotherapy. Urol Clin North Am 19:529-539.

Uvin P, Boudes M, Menigoz A, Franken J, Pinto S, Gevaert T, Verplaetse R, Tytgat J, Vennekens R, Voets T, et al. (2013) Chronic administration of anticholinergics in rats induces a shift from muscarinic to purinergic transmission in the bladder wall. Eur Urol 64:502-510.

Address correspondence to: Dr. Catherine McDermott, Faculty of Health Sciences and Medicine, Bond University, Robina, QLD 4229, Australia. E-mail: camcderm@bond.edu.au 